\title{
The miRNA-gene regulatory circuits in nSARS-CoV-2 and their potential correlations with pulmonary and thrombotic disorders
}

Pankaj Khurana ( $\sim$ pkhurana08@gmail.com )

DIPAS, DRDO

Apoorv Gupta

DIPAS, DRDO

Ragumani Sugadev

DIPAS, DRDO

Y K Sharma

DIPAS, DRDO

Rajeev Varshney

DIPAS, DRDO

Lilly Ganju

DIPAS, DRDO

Bhuvnesh Kumar

DIPAS, DRDO

\section{Research Article}

Keywords: miRNA regulatory networks, nSARS-CoV-2, COVID-19, Feed-Forward Loops, Network motifs, HAPE, Thrombosis

Posted Date: May 14th, 2020

DOI: https://doi.org/10.21203/rs.3.rs-28698/v1

License: (c) (1) This work is licensed under a Creative Commons Attribution 4.0 International License.

Read Full License 


\section{Abstract}

In view of the worldwide spread of the novel Severe Acute Respiratory Syndrome Coronavirus 2 (nSARSCoV-2) infection pandemic situation, research to repurpose drugs, identify novel drug targets, vaccine candidates, diagnostic markers etc have created a new race to curb the disease. To uncover nSARS-CoV2-related important biological features and understanding the molecular basis of this disease, network biology and miRNA-gene regulatory motif-based approach is used. 11 antiviral human-microRNAs (miRNAs) which can potentially target SARS-CoV-2 genes were collated; their direct miRNA interactors were identified and a comprehensive nSARS-CoV-2 responsive miRNA:Transcription Factor (TF):gene coregulatory network was built. 1385 miRNA:TF:gene tripartite, Feed-Forward Loops (FFLs) were identified from the network. The network topology was mapped into the biological space and the overrepresented pathways were identified. Four regulatory circuits: hsa-mir-9-5p-EP300-PLCB4, hsa-mir324-3p-MYC-HLA-F, hsa-mir-1827-E2F1-CTSV and hsa-mir-1277-5p-SP1-CANX are identified. These miRNA-gene regulatory circuits are found to regulate signalling pathways like virus endocytosis, viral replication, inflammatory response, pulmonary vascularization, cell cycle control, virus spike protein stabilization, antigen presentation, etc. Some novel computational evidences for understanding nSARSCoV-2 molecular mechanisms controlled by these regulatory circuits is put forth. The novel associations of miRNAs and genes identified with this infection are open for experimental validation. Further, these regulatory circuits also suggest potential correlations/similarity in the molecular mechanisms during nSARS-CoV-2 infection and pulmonary diseases and thromboembolic disorders. A detailed molecular snapshot of TGF- $\beta$ signalling pathway as the common mechanism that could play an important role in controlling common pathophysiology i.e. systemic inflammation, increased pulmonary pressure, ground glass opacities, D-dimer overexpression is also put forth.

\section{Introduction}

Coronaviruses are retroviruses that are sub-categorised in positive sense single stranded RNA viruses[1]. Their genomes size is of $\sim 30 \mathrm{~kb}$ that includes a 5'cap structure and a 3'polyA tail[2]. The different coronavirus can be further classified into four types: $\alpha, \beta, \gamma$, and $\delta$ based on specific genomic and protein regions[3]. nSARS-CoV- 2 belongs to the $\beta$ subtype of the coronavirus, which have $45-90 \%$ genetic similarity with the other $\beta$ subtype CoVs such as Severe Acute Respiratory Syndrome-Coronavirus (SARS$\mathrm{CoV}$ ) and Middle East Respiratory Syndrome coronavirus (MERS-CoV)[4]. The transcriptomic analysis of nSARS-COV-2 shows that some of these important proteins that are required for virus attachment, viral replication and pathogenesis are almost consistent in coronavirus with minute variations. These proteins are spike (S) glycoprotein, matrix (M) protein, small envelope (E) protein, and nucleocapsid $(N)$ protein. Spike glycoprotein (S) functions to bind virus with the host cell receptor, and mediate membrane fusion and virus entry[5]. S protein contains two subunits, $\mathrm{S} 1$ and $\mathrm{S} 2$, each is about $180 \mathrm{kDa}$ [6]. Recent reports suggested that $S$ protein of nSARS-CoV-2 has a strong interaction with human angiotensin-converting enzyme 2 (ACE2) especially that are expressed on lung alveolar epithelial cells [7]. The M (membrane) protein (also known as E1 membrane glycoprotein or matrix protein) functions together with the S (spike) 
and the $E$ (envelope) proteins. This protein is the crucial components of viral assembly and morphogenesis, involved in regulation of replication and packing the genomic RNA into viral particles [8]. Another viral protein called small envelope (E) protein having just 76 amino acids long, is membrane component of coronaviruses. This protein plays a significant role in coronavirus virion life cycle. Another important protein is nucleocapsid $(\mathrm{N})$ protein, which is a structural protein that forms complexes with genomic RNA [9]. The primary function of $\mathrm{N}$ protein is to interact with the viral membrane protein and enhance the efficiency of virus transcription and its assembly.

Recent findings have suggested that nSARS-CoV-2 can control expression of host regulatory molecules such as miRNAs and Transcription factor (TFs) $[10,11]$. Both of these regulatory biomolecules regulates the gene expression at transcriptional and post-transcriptional level [12]. Gene regulation is viewed as a complex process where regulatory elements and their targets form highly complex network interactions. Construction and analysis of miRNA:TF:gene coregulatory networks have been successfully implemented in many complex disease models like myocardial infarct, cancer, hypoxia etc to analyse multidimensional interactions to identify mechanistic insights and potential targets [13-15]. Motif identification and analysis have been extensively used to explore these regulatory networks. Network motifs are recurring short patterns in the network. One of the most overrepresented network motif is Feed Forward Loop (FFL) [16]. It is tripartite co-regulatory motif of a Transcription Factor (TF), miRNA and gene in which a TF regulates a miRNA or a miRNA regulates a TF, and both jointly coregulates a target gene. This three node motifs are found significantly overexpressed in gene regulatory networks [17]. Identification of these motifs have been found helpful in predicting important targets for diagnosis, prognosis and therapeutics purpose in multifactorial disorders [18]. Recent reports of nSARS-CoV-2 have correlated the pulmonary diseases and thrombosis in terms of similar pathology and symptoms[19-21]. These diseases also cause Acute Lung Injury (ALI) and Acute Respiratory Distress Syndrome (ARDS) that are very common in the critical patients of nSARS-CoV-2. The associations in these disease has lead to the possibility of repurposing of drugs that could be effective for treating critical patients[22]. Though there are many published reports on associations in clinical findings or relationships among their pathology and symptoms but similarity in molecular mechanisms is still not well studied. Analysis of the complex regulatory interactions of these antiviral human miRNAs and their interactions have been used to identify relationships between these diseases and nSARS- CoV-2 at molecular level.

11 antiviral human-microRNAs (miRNAs) which can potentially target SARS-CoV-2 genes were collated [10]. Direct miRNA interactors for these are identified and a comprehensive nSARS-CoV-2 responsive miRNA:TF:gene coregulatory network was built. Overrepresented biological pathways and regulatory FFLs are analysed and a possible molecular mechanism and checkpoints are proposed. Further these circuits provide insights about nSARS-CoV-2 infection similarity with other pulmonary diseases and thromboembolic disorders with interconnections at molecular level.

\section{Methodology}




\section{Identification of miRNAs associated with nSARS-CoV-2 antiviral host-miRNAs}

The list of 11 antiviral human-microRNAs (miRNAs) which can potentially target SARS-CoV- 2 genes were extracted[10] . Their direct miRNA interactors were identified from PmmR database [23]. PmmR is a database of putative miRNA-miRNA interactions identified by analysing miRNA:TF: inter-regulatory networks with the help of shortest path length as a scoring function. To make the results more stringent, interactions having score $>0.7$ were only considered. This yielded an exhaustive list of 58 miRNAs which was further used to identify target genes.

\section{Construction of miRNA:TF:gene co-regulatory network}

The miRNA:target genes were identified using the MultimiR R package[24]. The package collects data from several validated databases, including mirTarBase, miRecords and tarbase etc. A comprehensive list of all human TFs was collated from several databases i.e. TFcheckpoint[25], DBD[26], ORFeome[27], TcoF-DB V2[28], TFCat[29],

TFClass[30]TRANSFAC [31]. The target genes were compared with this comprehensive TF list and those matching were labelled as TFs and others were labelled as genes. Thus the resultant miRNA:target gene interactions were also annotated as miRNA:gene or miRNA:TF interactions. TF:gene and TF:miRNA interactions were also added from respective public repositories. The TF:gene interactions were fetched from oregAnno 3.0 [32] and TRRUST V2[33] databases. TF: miRNA interactions were fetched from TransmiR[34] and PuTmiR[35] databases. Finally a comprehensive nSARS-CoV-2 responsive miRNA: TF :gene coregulatory network was built.

\section{Pathway Enrichments}

Reactome pathway analyser is a powerful tool to mine signalling pathways of genes sets [36]. This was used to identify the enriched pathways in the TFs and genes of nSARS-CoV-2 responsive miRNA:TF:gene regulatory network. Further the enriched pathways were analysed by boxplot for identification of outliers.

\section{Identification of miRNA:TF:gene co-regulatory FFL motifs.}

Among the various types of motifs that can be potentially identified from a network, a randomization test was performed to evaluate the significance of the FFLs. For this, how often one FFL appears in the real network to the number of times it appears in randomly generated networks formed by degree preserving randomization algorithm is compared. In order to retain biological key driver nodes, a degree preserving randomization algorithm of the 'igraph' $R$ - package is used [37](). Randomization process is repeated 100 times. Z score for each motif type was calculated to identify the significant motifs. 
No is the number of motifs observed in the real network, whereas $\mathrm{Nm}$, and $\sigma$ are the mean and standard deviation of the motif occurrence in 100 random networks, respectively.

Thereafter, FFLs were identified from miRNA:TF:gene coregulatory network module using in-house python scripts based on graph theory principle.

\section{Results}

Forty-seven miRNAs interacting with 11 antiviral host-miRNAs are identified using the PmmR database (Supplemntary Table S1). 5,000 experimentally validated gene targets of these 58 human miRNAs were identified. These targets were annotated as TFs or genes by comparing them with a comprehensive list human TFs. Hence miRNA:target interactions were categorised as miRNA:TF and miRNA:gene interactions. Additionally TF:gene, TF:miRNA interactions were added to construct the comprehensive nSARS-CoV-2 responsive miRNA:TF:gene coregulatory network. This network contains 58 miRNAs, 300 TFs, 4696 genes. (Supplementary Figure S1).

\section{Pathway Enrichment}

The TF and genes in the comprehensive nSARS-CoV-2 responsive miRNA:TF:gene coregulatory network were subjected to pathway enrichment using the Reactome pathway analyser [36]. 68 pathways having $p$ value $<0.1$ were identified (Supplementary Table S2). To identify the most enriched pathways in the network, these pathways terms were distributed based on modulus of log2 (pValue) using boxplot (Figure 1).

"Endosomal/Vacuolar pathway" and "Antigen Presentation: Folding, assembly, and peptide loading for MHC class 1" were identified as outliers. Hence TFs and genes in these pathways were chosen for further analysis.

In a parallel study, a list of 81 genes related to COVID-19, SARS, MERS were curated from literature by text-mining and manual curation (Supplementary Table S3). Further their associated regulatory miRNAs, TFs and related interaction were retrieved and an analogous miRNA:TF:gene coregulatory network was constructed. Pathway enrichment analysis of this network also shows "Endosomal/Vacuolar signalling pathway" and "Antigen Presentation: Folding, assembly, and peptide loading for MHC class 1" as enriched pathways (supplementary Table S4). This cross-validates the results i.e. if we begin with set host antiviral miRNAs or a nSARS-CoV-2 genelist, these two pathways are the most enriched.

\section{FFL Analysis}


Network motifs are simple-subgraphs recur in complex networks. Four different subgraph (motifs) were identified in the nSARS-CoV-2 responsive miRNA:TF:gene coregulatory network. Randomization was performed for 100 random network to calculate the Z-score for each type of subgraph. Randomization of network shows motif 000100110 have highest Z- score of -0.907 (Supplementary Table S5). The 000100110 type subgraph are FFLs motifs that were selected for further evaluation. FFLs are statistically over-represented tri-partite motifs that play crucial regulatory roles in biological networks. $1385 \mathrm{FFLs}$ were identified and a resultant network contained 17 miRNAs, 101 TFs, 84 gene targets (Figure 2).

Four of these FFLs, contained TFs and genes associated with "Endosomal/Vacuolar pathway" and "Antigen Presentation: Folding, assembly, and peptide loading for MHC class 1". These FFLs are A: hsamir-9-5p-EP300-PLCB4, B: hsa-mir-324-3p-MYC-HLA-F, C: hsa-mir-1827- E2F1-CTSV and D: hsa-mir-12775p-SP1-CANX (Table 1). FFL $A$ and $B$ are common to both the pathways; whereas FFL $C$ and $D$ are unique to "Endosomal/Vacuolar pathway" and "Antigen Presentation: Folding, assembly, and peptide loading for MHC class 1" respectively.

\begin{tabular}{|r|l|l|l|l|}
\hline FFL & miRNA & TF & Gene & Pathways \\
\hline A & $\begin{array}{l}\text { hsa-mir-9- } \\
5 p\end{array}$ & EP300 & PLCB4 & $\begin{array}{l}\text { Endosomal/Vacuolar pathway, Antigen } \\
\text { Presentation: Folding, assembly, and peptide } \\
\text { loading for MHC class 1 }\end{array}$ \\
\hline B & $\begin{array}{l}\text { hsa-mir- } \\
324-3 p\end{array}$ & MYC & HLA-F & $\begin{array}{l}\text { Endosomal/Vacuolar pathway, Antigen } \\
\text { Presentation: Folding, assembly, and peptide } \\
\text { loading for MHC class 1 }\end{array}$ \\
\hline C & $\begin{array}{l}\text { hsa-mir- } \\
1827\end{array}$ & E2F1 & CTSV & Endosomal/Vacuolar pathway \\
\hline D & $\begin{array}{l}\text { hsa-mir- } \\
1277-5 p\end{array}$ & SP1 & CANX & $\begin{array}{l}\text { Antigen Presentation: Folding, assembly, and } \\
\text { peptide loading for MHC class 1 }\end{array}$ \\
\hline
\end{tabular}

Table 1: The FFL motifs identified in nSARS-CoV-2 responsive miRNA:TF:gene coregulatory network having its target genes or TFs in the enriched pathways.

\section{Discussion}

TFs and miRNAs are the two most important key regulators of gene expression at the transcriptional and posttranscriptional levels. Evidence from the literature has suggested the importance of their complex combinatorial regulation in various cellular systems and diseases. miRNAs and TFs are part of a complex combinatorial regulatory mechanism that can be seen in the miRN:TF:gene coregulatory network. These combinatorial regulations can be easily understood by studying miRNA, TF, gene tripartite motifs known as FFLs which are overrepresented in their coregulatory networks [38, 39]. The analysis was done on nSARS- CoV-2 biomolecules. The pathway enrichment analysis of nSARS-CoV-2 responsive miRNA:TF:gene coregulatory network constructed using 58 miRNAs shows that "Endosomal/Vacuolar signalling" pathway and "Antigen Presentation: Folding, assembly, and peptide loading for MHC class 1" are most enriched pathways. Endosomal/vacuolar pathway, also known as Endocytic Pathway, has been recently reported as major targeting pathway for designing COVID-19 therapeutics because it controls key 
element in viral infection i.e. process of viral entry into the host cells[40]. The endocytic pathway including endosome and lysosome has been used for development of therapeutic strategies in combating diseases caused by other CoVs i.e, SARS, MERS[40]. Additionally pathway enrichment analysis of miRNA:TF:gene coregulatory network constructed using 81 genes of SARS, MERS, COVID-19 genes also shows "Endosomal/vacuolar pathway" as most enriched pathway in the network. Similarly second most enriched pathway in the network namely "Antigen Presentation: Folding, assembly, and peptide loading for MHC class 1" pathway is reported to effect the susceptibility and severity of nSARSCoV-2 virus infection in the patients. [41]. Hence genes sets of these two pathways were considered for further analysis.

The FFL analysis of miRNA:TF:gene coregulatory identified 1385 FFLs. Out of these FFLs, four FFLs contained TF and genes belonging to the enriched pathways. These FFLs are A: hsa- mir-9-5p-EP300PLCB4, B: hsa-mir-324-3p-MYC-HLA-F, C: hsa-mir-1827-E2F1-CTSV and D: hsa-mir-1277-5p-SP1-CANX. These are referred to as miRNA-gene regulatory circuits. Although these FFLs do not have any common nodes but there are many interconnections between them for e.g. FFL $A$ is connected to FFL B through EP300 regulating MYC and vice- versa. Similarly FFL C is connected to FFL B through both E2F1, hsamiR-1827 regulating MYC. (Figure 3). This closed circuit is known to regulate/control important biological processes as shown in Figure 3 and discussed below.

The FFL A: hsa-mir-9-5p-EP300-PLCB4 contains miRNA- hsa-miR-9-5p coregulating TF- EP300 and genePLCB4. Hsa-miR-9-5p was recently reported to target the 3' UTR of Angiotensin-converting enzyme 2 (ACE2)[42]. ACE2 receptor is identified as the main receptor that binds to the spike protein of the COVID19 virus and triggers viral RNA endocytosis[43]. It is also identified an initiator of pro-inflammatory cytokine production, and type I interferon production during SARS-CoV infection [44]. Hence hsa-miR-9-5p can be used as a potential target to regulate endocytosis of nSARS-CoV-2 viral RNA and uncontrolled lung inflammation. In FFL A: hsa-miR-9-5p is found to regulats E1A Binding Protein P300 (EP300), a known transcriptional co-activator protein of Hypoxia-Inducible Factor-1(HIF-1)

[45] and stimulator of Vascular Endothelial Growth Factor (VEGF) [46]. VEGF is already identified as a vascular permeability inducers in severe or critical patients with COVID-19 pneumonia. So an anti-VEGF drug Bevacizumab is already in the clinical trial to treat critical patients of COVID-19 with ClinicalTrials.gov Identifier: NCT04275414. Both of these miRNA and TFs regulates a common target known as Phospholipase C Beta 4 (PLCB4) in the FFL A. PLCB4 function in intracellular transduction of many extracellular signals. But there are no reports of association between PLCB4 and nSARS-CoV-2, SARS and MERS diseases. As PLCB4 is under tight regulation of hsa-miR-9-5p and EP300, that regulate important molecular functions during nSARS-CoV-2 infection, it might be important novel target for studying its role in nSARS-CoV-2. FFL B hsa-mir-324-3p-MYC-HLA-F contains hsa-miR-324-3p as major regulator. Hsa-miR-324-3p was found to inhibit viral replication of RNA viruses like influenza and SARS in host cells by targeting its (Protein Binding 1 to viral RNA) PB1[47]. In FFL B, hsa-miR-324-3p deregulates MYC which is a known transcription factor and oncogene controlling cell cycle progression, cell proliferation, and apoptosis [48]. MYC activation also induces the activation of PI3K-AKT pathway. PI3K 
-AKT pathway was found important pathway that controls virus infection host-cell proliferating genes and possibly helping in viral replication.[49, 50]. FFL analysis shows hsa-mir-324-3p and MYC coregulates the HLA-F gene expression. (Human leukocyte antigen) HLA proteins are found as susceptible antigens for nSARS-CoV-2 virus in human leukocytes [41]. Reports also show MYC and hsamiR-324-3p have inverted expression profile compared with HLA proteins [51]. This shows HLA-F expression can be regulated by both MYC and hsa-mir-324-3p in human leukocytes and targeting it can enhance the efficacy of the vaccine designed against nSARS-CoV-2. Hence this tightly regulated module where all biomolecules are important during SARS infection could be studied in detail experimentally. miRNAs and TFs of other two unique FFLs: hsa- mir-1827-E2F1- CTSV and hsa-mir-1277-5p- SP1- CANX are also found to regulate two important genes Cathepsin V (CTSV) and Calnexin (CANX). Both of these proteins binds to Spike protein of coronaviruses of nSARS-CoV-2 and regulate virus entry and stabilizes spike protein for its folding in host cell $[6,52]$. The regulatory transcription factor of FFL C and FFL D are SP1 and E2F1. SP1 was reported to trans-activate the TGF-B1 protein in lung tissues during SARS-CoV infection[53]. Increase in the level of TGF-B1 protein in plasma and lungs during SARS-CoV infection gives rise to tissue fibrosis in lungs. E2F1 was found to downregulate the S-phase genes expression in SARS-CoV infected lung tissue that promotes cell cycle progression and virus replication[54]. As both the mechanisms were also found in nSARS-CoV-2, inhibiting E2F1 can weaken the rate of virus replication. Hsa-mir-1827 and hsa-mir-1277-5p are the regulators of FFL C and FFL D. Hsa-mir-1827 was found to regulate tumor suppression in lung carcinomas[55]. There is no evidence of hsa-mir-1277-5p in any pulmonary disease; but they regulate important TFs and genes in the FFLs identified during this analysis. Hence both these miRNAs are proposed as novel targets and open for future experimental analysis and validation. This miRNA-gene circuit with their literature insights have been illustrated in (Figure 3).

Clinical finding of critical nSARS-CoV-2 patients mainly admitted to intensive care units shows consistent high level proinflammatory cytokines in their plasma $[56,57]$. This cytokine storm was found responsible for causing systemic inflammation that leads to the activation of the too many immune cells such as macrophages, lymphocytes [57]. The increase in the level of cytokines and systematic inflammation were found as the trigger for lung inflammation and coagulation in nSARS-CoV-2 patients [58]. The common symptoms shows activation of cytokine storm with consistent fever, cytopenia, enlarged liver or spleen etc[59] . The variety of cytokines activated during nSARS-CoV-2 infection and the most frequent are interleukins IL2, IL-2R, IL-6, IL1RN, IL-10, IL-7 etc.[56, 60]. Some of these interleukins IL6, IL10, and IL1RN have been also found in the FFLs of nSARS-CoV-2 responsive miRNA:TF: gene coregulatory network (Supplementary Table S7). These interleukins were found to be co- regulated by miRNA, TF i.e hsa-mir1277-5p, hsa-mir-98-5p, hsa-mir-149-3p, hsa-mir-125a- 5p, hsa-mir-9-5p, hsa-mir-23b-3p, hsa-mir-365a-3p and TFs EP300, FOXO1, PPARA, REL, respectively.

Other inflammatory cytokines like TNF- $\alpha$, TGF- $\beta$ that have been found correlated with coagulation and systematic inflammation during nSARS-CoV-2 are also important signalling molecules of other pathologies i.e lung injury and Thrombosis[61]. miRNA, TF regulators of these inflammatory cytokines found in the nSARS-CoV-2 responsive miRNA:TF:gene coregulatory network have also been found to be 
highly correlated with nSARS-CoV-2 and their potential correlations with pulmonary diseases and coagulation (Figure 4, Figure 5).

Recent reports show that nSARS-CoV infection has a symptom similarity with some of the pulmonary diseases like ALI, ARDS, and Chronic Pulmonary Obstructive Disorder (COPD) [62, 63]. This hints towards the possibility of cohesion in terms of molecular signalling and pathology of pulmonary diseases with nSARS-CoV-2. But there are some contrasting reports for establishing comorbidity in HAPE and nSARSCoV-2. Some reports favour the similarity in their pathophysiology's [19]. Whereas other reports decline the similarity as HAPE is non- cardiogenic form of pulmonary edema caused due to hypoxic pulmonary vasoconstriction[64] and support that nSARS-CoV-2 caused lung injury is only due to viral mediated inflammation[64]. Both the studies might be true but they lack the support of molecular evidence. Hence we try to overlay our FFL analysis results and literature finding over well- established HAPE framework (Figure 4).

For this a list of 93 genes related to HAPE was manually curated from the literature (Supplementary Table S6). Six of these genes/TFs are also present in the FFL network of nSARS-CoV-2. These include PPARA, MAP2K7, IL6, IL10, IL1RN and VCAM1. These genes are linked to HAPE as biomarker or their polymorphism as a risk factor in HAPE- resistive Vs HAPE susceptible patients [65-67]. When these 6 genes were mapped on the FFLs, 15 FFLs were identified and found that most of the miRNAs regulated the TGF-beta signalling and tumor suppression in pulmonary diseases (Supplementary table S7). Also, the plasma levels of IL6, IL10 and IL1RN were found increased in the critical nSARS-CoV-2 patients[57]. So systemic inflammation caused due to strong influx of cytokines like interleukins has strong association between pulmonary diseases like HAPE and nSARS-CoV-2. Literature also shows increased pulmonary pressure and ground glass opacities were found common in both HAPE and nSARS-CoV-2 patients [19]. During HAPE, hypobaric hypoxia causes decrease in arterial oxygen leading to the pulmonary vasoconstriction and further leads to increase in pulmonary pressure. TGF- $\beta$ signalling was identified to control pulmonary pressure in lungs and TGF- B1 and TGFBR receptors-1,2 complex are the major regulators of the signalling [68].Sustained increase in pulmonary pressure causes pulmonary hypertension, over perfusion that further leads to pulmonary vascular leakage causing edema. Recent published reports also shows that TGF-beta1 appear to be overproduced in COVID-19 patients [69]. HsamiR-9-5p, hsa-miR- 98-5p and hsa-miR-23b-3p regulate TGFBR receptors during pulmonary disorders [68, $70,71]$. These miRNAs are part of the regulatory circuit identified in the present study and are antiviral host-miRNAs which can potentially target SARS-CoV-2 genes [10]. Also pulmonary hypertension impairs nitrous oxide synthesis that induces RAS pathways proteins during HAPE. These proteins includes IL6, ACE, ACE2 that cause inflammation and over-perfusion leading to pulmonary vascular leakage[72]. ACE2 and its receptors have been widely identified as risk factor for COVID-19 patients. Also decrease in arterial oxygen during HAPE leads to decrease in intercellular $\mathrm{Ca}++$ homeostasis marked with ROS generation causing loss of endothelial cellular integrity. This induces tissue necrosis in lungs seen as ground glass opacities in radiological scans of HAPE and lung carcinoma patients [73, 74]. This loss of endothelial cellular integrity causing necrosis is also controlled by TGF-B signalling $[75,76]$. The ground glass opacities is reported in both nSARS-CoV-2 infection and lung carcinomas. miRNAs hsa-miR-149-5p, hsa- 
miR-125a-5p, hsa-miR-1827 in our 15 FFLs were found to regulate tumor suppression cell migration, invasion and apoptosis in the lung carcinomas with lesion formation.[77, 78]. Hence these miRNAs can be studied and their role in tissue necrosis in nSARS-CoV-2 infection can be explored. The literature also highlights commonality in pathophysiology like pulmonary pressure, lung fibrosis in both HAPE and SARS-CoV viral infection $[53,68]$. This factor is important in repurposing of drugs like Acetazolamide, Nifedipine and Phosphodiesterase inhibitors that have been proposed to be repurposed for COVID-19 [19]. Acetazolamide has a myriad of effects on different organ systems, potently reduces hypoxic pulmonary vasoconstriction and improves minute ventilation; whereas Nifedipine and Phosphodiesterase inhibitors are directed to decrease pulmonary pressure in HAPE patients[19].

The miRNAs proposed in the current study were submitted to Pharmaco-miR[79] server to identify their potential interactions with drugs. Nifedipine and Acetazolamide were found to be associated with the expression of hsa-mir-98 and hsa-mir-23b respectively [79]. Further screening of other miRNA, TF, gene targets in network can also be experimentally validated to design therapeutic drugs, diagnosis marker, prognosis marker or vaccine against nSARS- CoV-2.

Recent observations of nSARS-CoV-2 cases also suggest that, pulmonary and lung injury in COVID-19 is not only factor to drive patient to acute respiratory distress syndrome (ARDS) and further to Acute cardiac injury (ACl), Acute Kidney injury(AKI) and multiple organ failure. Other factors like thrombosis and sepsis are also proposed [80]. Activation of coagulation can be easily found in variety of virus infections like retrovirus outbreak including HIV, Dengue virus, and Ebola virus especially in SARS outbreak of 2003[81]. Reports suggested that high morbidity and mortality rates of SARS which is closely related to nSARSCoV-2 is due to the vascular endothelial damage together with Disseminated Intravascular Coagulation (DIC), Deep Vein Thrombosis (DVT) and Pulmonary Embolism (PE) that leads to the pulmonary infarction [82, 83]. Increase of D-dimers in nSARS-CoV-2 patients during nSARS-CoV-2 disease progression with chest $\mathrm{CT}$ shows strong association with venous thrombosis and pulmonary embolism [60]. D-dimers are the product of degraded cross-linked fibrin from the clot formation [84]. Whether molecular mechanism leading to clot can be virus mediated or result of systemic inflammation is the grey area of research.

The clot formation is initiated in the most type of lung injuries[85]. Lung injury leads to the formation fibrin gels inside the alveolar compartment. The fibrin gel is the accumulation of tissue fibrins formed after disruption of fibrous network around human lung fibroblasts. Due to the lack of fibrin network around human lung fibroblasts, they escape the cell matrix and support the organization of these fibrin gels. This growth of fibrin gels further leads to clot formation. After clot formation, fibrinolysis is a process that prevents blood clots from growing. During fibrinolysis, plasminogen is reduced to plasmin by tissue plasminogen activator (tPA) and urokinase (UPA) (Figure 5).

Plasmin than degrades the clot fibrin network into smaller products and reduces further clot formation [86]. HLF plasminogen activator inhibitor-1 (PAI-1) or SERPINE1 is identified as inhibitor of tPA and uPA. Inhibition of tPA and uPA leads to the inactivation of plasmin enzyme that further prevents the process of 
fibrinolysis [86]. Literature shows lung injury also induces cytokines like transforming growth factor-beta (TGF- $\beta$ ) and tumor necrosis factor- alpha (TNF-alpha). TGFB1 and TNF-alpha has been extensively studied in Human Lung Fibroblasts (HLF) for their role in fibrinolytic and procoagulant activities. TGFB1 help in the induction HLF plasminogen activator inhibitor-1 (PAI-1), Human Coagulation Factor XII in human lung fibroblast and TNF- $\alpha$ induces release of PAI-1 from the cells and inactivates tPA. [86, 87]. This way, TGF- $\beta$ and TNF- $\alpha$ impair the ability of HLF to degrade clot fibrin by disturbing the balance of HLF plasminogen activators and PAI-1. These pathways may therefore be targeted for a possible mechanism to protect patient from pulmonary embolism and thrombosis.

It was found that nucleocapsid $(\mathrm{N})$ protein potentiates TGF- $\beta$-induced expression of PAI-1 during SARSCoV infection[88]. The PAI-1 or SERPINE1 is regulated by many TFs in SARS- CoV-2 responsive miRNA: TF:gene coregulatory network (supplementary Table 8). Some of these TFs i.e SMAD-3,SMAD4, EP300 have been found modulated by $\mathrm{N}$ protein of SARS- CoV virus [88]. $\mathrm{N}$ protein binds to SMAD3/SMAD4 of host cell and promotes SMAD3-EP300 complex formation that regulates the TGF- $\beta$ signalling pathway during SARS-CoV infection [88]. So hsa-miR-9-5p that dysregulates the EP300 in the proposed miRNA-TFgene regulatory circuit can also be used as target in nSARS-CoV-2[89]. Interleukin IL6 induction was also seen in the later stage nSARS-CoV-2 patients [60]. It was also seen that IL-6 has opposite expression profile as compared with D-dimer in SARS-CoV2 patients. Its elevation start from 13 days onwards of the infection as when D-dimers start to decrease [60]. IL-6 promotes coagulation without affecting fibrinolysis [90]. Hence increase in IL6 and decrease in D-dimer might be an indicator of inhibition of fibrinolysis, which can further leads to the growth of clot to the extent causing serious thrombotic conditions.

Our study thus shows the relation of nSARS-CoV-2 with clotting, pulmonary embolism with molecular signalling involving fibrinolysis as major pathway that increases the D-dimers in the SARS-CoV-2 infection. Cytokines TGF- $\beta$ and TNF- $\alpha$ are proposed as the major regulator of fibrinolysis controlling proteins PAI-1 and plasminogen activators found in SARS infection.

\section{Declarations}

Competing interests: The authors declare no competing interests.

\section{References}

1. Schoeman D, Fielding BC. Coronavirus envelope protein: current knowledge. Virology journal. 2019;16:69.

2. Yang D, Leibowitz JL. The structure and functions of coronavirus genomic 3 ' and 5 ' ends. Virus research. 2015;206:120-33.

3. Fehr AR, Perlman S. Coronaviruses: an overview of their replication and pathogenesis. Methods in molecular biology. 2015;1282:1-23.

4. Zheng J. SARS-CoV-2: an Emerging Coronavirus that Causes a Global Threat. International journal of biological sciences. 2020;16:1678-85.

Page $11 / 20$ 
5. Mousavizadeh L, Ghasemi S. Genotype and phenotype of COVID-19: Their roles in pathogenesis. Journal of microbiology, immunology, and infection = Wei mian yu gan ran za zhi.

6. Ou X, Liu Y, Lei X, Li P, Mi D, Ren L, et al. Characterization of spike glycoprotein of SARS-CoV-2 on virus entry and its immune cross-reactivity with SARS-CoV. Nature 2020;11:1620.

7. Zhang $\mathrm{H}$, Penninger JM, Li Y, Zhong $\mathrm{N}$, Slutsky AS. Angiotensin-converting enzyme 2 (ACE2) as a SARS-CoV-2 receptor: molecular mechanisms and potential therapeutic target. Intensive care medicine. 2020;46:586-90.

8. Hu Y, Wen J, Tang L, Zhang H, Zhang X, Li Y, et al. The M protein of SARS-CoV: basic structural and immunological properties. Genomics, proteomics \& bioinformatics. 2003;1:118-30.

9. McBride R, van Zyl M, Fielding BC. The coronavirus nucleocapsid is a multifunctional protein. Viruses. 2014;6:2991-3018.

10. Rahila Sardar DS, Shweta Birla, Dinesh Gupta. Comparative analyses of SAR-CoV2 genomes from different geographical locations and other coronavirus family genomes reveals unique features potentially consequential to host-virus interaction and pathogenesis. bioRxiv

11. Müşerref Duygu Saçar Demirci Computational analysis of microRNA-mediated interactions in SARSCoV-2 infection. BioRxiv. 2020.

12. Martinez NJ, Walhout The interplay between transcription factors and microRNAs in genome-scale regulatory networks. BioEssays : news and reviews in molecular, cellular and developmental biology. 2009;31:435-45.

13. Lin Y, Sibanda VL, Zhang HM, Hu H, Liu H, Guo AY. MiRNA and TF co-regulatory network analysis for the pathology and recurrence of myocardial infarction. Scientific reports. 2015;5:9653.

14. Gupta A, Sugadev R, Sharma YK, Yahmad Y, Khurana P. Role of miRNAs in hypoxia-related disorders. Journal of biosciences. 2018;43:739-49.

15. Li R, Chen H, Jiang S, Li W, Li H, Zhang Z, et CMTCN: a web tool for investigating cancer-specifiC microRNA and transcription factor co-regulatory networks. PeerJ. 2018;6:e5951.

16. Mangan S, Alon U. Structure and function of the feed-forward loop network motif. Proceedings of the National Academy of Sciences of the United States of America. 2003;100:11980-5.

17. Hsieh WT, Tzeng KR, Ciou JS, Tsai JJ, Kurubanjerdjit N, Huang $\mathrm{CH}$, et al. Transcription factor and microRNA-regulated network motifs for cancer and signal transduction networks. BMC systems biology. 2015;9 Suppl 1:S5.

18. Wang L, Zhao H, Li J, Xu Y, Lan Y, Yin W, et al. Identifying functions and prognostic biomarkers of network motifs marked by diverse chromatin states in human cell lines. Oncogene. 2020;39:677-89.

19. Solaimanzadeh Acetazolamide, Nifedipine and Phosphodiesterase Inhibitors: Rationale for Their Utilization as Adjunctive Countermeasures in the Treatment of Coronavirus Disease 2019 (COVID19). Cureus. 2020;12:e7343.

20. Han H, Yang L, Liu R, Liu F, Wu KL, Li J, et al. Prominent changes in blood coagulation of patients with SARS-CoV-2 infection. Clinical chemistry and laboratory medicine. 
21. Connors JM, Levy JH. COVID-19 and its implications for thrombosis and anticoagulation. Blood. 2020.

22. Bing He LG. Prediction of repurposed drugs for treating lung injury in COVID-19. arXivorg.

23. Sengupta $D$, Bandyopadhyay S. Participation of microRNAs in human interactome: extraction of microRNA-microRNA regulations. Molecular bioSystems. 2011;7:1966-73.

24. Ru Y, Kechris KJ, Tabakoff B, Hoffman P, Radcliffe RA, Bowler R, et The multiMiR R package and database: integration of microRNA-target interactions along with their disease and drug associations. Nucleic acids research. 2014;42:e133.

25. Chawla K, Tripathi S, Thommesen L, Laegreid A, Kuiper M. TFcheckpoint: a curated compendium of specific DNA-binding RNA polymerase II transcription factors. Bioinformatics. 2013;29:2519-20.

26. Kummerfeld SK, Teichmann SA. DBD: a transcription factor prediction database. Nucleic acids research. 2006;34:D74-81.

27. Lamesch P, Li N, Milstein S, Fan C, Hao T, Szabo G, et al. hORFeome v3.1: a resource of human open reading frames representing over 10,000 human genes. Genomics. 2007;89:307-15.

28. Schaefer U, Schmeier S, Bajic VB. TcoF-DB: dragon database for human transcription co-factors and transcription factor interacting proteins. Nucleic acids research. 2011;39:D106-10.

29. Fulton DL, Sundararajan S, Badis G, Hughes TR, Wasserman WW, Roach JC, et al. TFCat: the curated catalog of mouse and human transcription factors. Genome biology. 2009;10:R29.

30. Wingender E, Schoeps T, Haubrock M, Krull M, Donitz J. TFClass: expanding the classification of human transcription factors to their mammalian Nucleic acids research. 2018;46:D343-D7.

31. Matys V, Fricke E, Geffers R, Gossling E, Haubrock M, Hehl R, et al. TRANSFAC: transcriptional regulation, from patterns to profiles. Nucleic acids research. 2003;31:374-8.

32. Lesurf R, Cotto KC, Wang G, Griffith M, Kasaian K, Jones SJ, et al. ORegAnno 3.0: a communitydriven resource for curated regulatory annotation. Nucleic acids research. 2016;44:D126-32.

33. Han H, Cho JW, Lee S, Yun A, Kim H, Bae D, et al. TRRUST v2: an expanded reference database of human and mouse transcriptional regulatory interactions. Nucleic acids research. 2018;46:D380-D6.

34. Tong Z, Cui Q, Wang J, Zhou Y. TransmiR 0: an updated transcription factor-microRNA regulation database. Nucleic acids research. 2019;47:D253-D8.

35. Bandyopadhyay S, Bhattacharyya PuTmiR: a database for extracting neighboring transcription factors of human microRNAs. BMC bioinformatics. 2010;11:190.

36. Croft D, O'Kelly G, Wu G, Haw R, Gillespie M, Matthews L, et Reactome: a database of reactions, pathways and biological processes. Nucleic acids research. 2011;39:D691-7.

37. Iorio F, Bernardo-Faura M, Gobbi A, Cokelaer T, Jurman G, Saez-Rodriguez J. Efficient randomization of biological networks while preserving functional characterization of individual BMC bioinformatics. 2016;17:542.

38. Yan Z, Shah PK, Amin SB, Samur MK, Huang N, Wang X, et al. Integrative analysis of gene and miRNA expression profiles with transcription factor-miRNA feed-forward loops identifies regulators in 
human cancers. Nucleic acids research. 2012;40:e135.

39. Shen-Orr SS, Milo R, Mangan S, Alon U. Network motifs in the transcriptional regulation network of Escherichia coli. Nature genetics. 2002;31:64-8.

40. Yang N, Shen Targeting the Endocytic Pathway and Autophagy Process as a Novel Therapeutic Strategy in COVID-19. International journal of biological sciences. 2020;16:1724-31.

41. Nguyen A, David JK, Maden SK, Wood MA, Weeder BR, Nellore A, et Human leukocyte antigen susceptibility map for SARS-CoV-2. Journal of virology. 2020.

42. Chen LZ, L. Lung Adenocarcinoma Patients Own Higher Risk of SARS-CoV-2 Infection. Preprint. 2020.

43. Hussain M, Jabeen N, Raza F, Shabbir S, Baig AA, Amanullah A, et Structural variations in human ACE2 may influence its binding with SARS-CoV-2 spike protein. Journal of medical virology. 2020.

44. Leon-Icaza SA, Zeng M, Rosas-Taraco microRNAs in viral acute respiratory infections: immune regulation, biomarkers, therapy, and vaccines. ExRNA. 2019;1:1.

45. Wei J, Yang Y, Lu M, Lei Y, Xu L, Jiang Z, et al. Recent Advances in the Discovery of HIF-1alphap300/CBP Inhibitors as Anti-Cancer Agents. Mini reviews in medicinal chemistry. 2018;18:296-309.

46. Fish JE, Cantu Gutierrez M, Dang LT, Khyzha N, Chen Z, Veitch S, et Dynamic regulation of VEGFinducible genes by an ERK/ERG/p300 transcriptional network. Development. 2017;144:2428-44.

47. Kumar A, Kumar A, Ingle H, Kumar S, Mishra R, Verma MK, et al. MicroRNA hsa-miR-324-5p Suppresses H5N1 Virus Replication by Targeting the Viral PB1 and Host CUEDC2. Journal of virology. 2018;92.

48. McMahon SB. MYC and the control of apoptosis. Cold Spring Harbor perspectives in medicine. 2014;4:a014407.

49. Bretones G, Delgado MD, Leon J. Myc and cell cycle control. Biochimica et biophysica acta. 2015;1849:506-16.

50. Zhu J, Blenis J, Yuan J. Activation of PI3K/Akt and MAPK pathways regulates Myc-mediated transcription by phosphorylating and promoting the degradation of Proceedings of the National Academy of Sciences of the United States of America. 2008;105:6584-9.

51. Peltenburg LT, Schrier PI. Transcriptional suppression of HLA-B expression by c-Myc is mediated through the core promoter elements. Immunogenetics. 1994;40:54-61.

52. Fukushi M, Yoshinaka Y, Matsuoka Y, Hatakeyama S, Ishizaka Y, Kirikae T, et al. Monitoring of S protein maturation in the endoplasmic reticulum by calnexin is important for the infectivity of severe acute respiratory syndrome coronavirus. Journal of virology. 2012;86:11745-53.

53. Li SW, Wang CY, Jou YJ, Yang TC, Huang SH, Wan L, et al. SARS coronavirus papain-like protease induces Egr-1-dependent up-regulation of TGF-beta1 via ROS/p38 MAPK/STAT3 pathway. Scientific reports. 2016;6:25754.

54. Surjit M, Liu B, Chow VT, Lal SK. The nucleocapsid protein of severe acute respiratory syndromecoronavirus inhibits the activity of cyclin-cyclin-dependent kinase complex and blocks $S$ phase 
progression in mammalian cells. The Journal of biological chemistry. 2006;281:10669-81.

55. Li Y, Yin Z, Fan J, Zhang S, Yang W. The roles of exosomal miRNAs and IncRNAs in lung diseases. Signal transduction and targeted therapy. 2019;4:47.

56. Chen G, Wu D, Guo W, Cao Y, Huang D, Wang H, et al. Clinical and immunological features of severe and moderate coronavirus disease 2019. The Journal of clinical investigation. 2020;130:2620- 9.

57. Mehta P, McAuley DF, Brown M, Sanchez E, Tattersall RS, Manson JJ, et al. COVID-19: consider cytokine storm syndromes and immunosuppression. Lancet. 2020;395:1033-4.

58. Zhou F, Yu T, Du R, Fan G, Liu Y, Liu Z, et al. Clinical course and risk factors for mortality of adult inpatients with COVID-19 in Wuhan, China: a retrospective cohort study. Lancet. 2020;395:1054-62.

59. Zhang Z, Wang J, Ji B, Bahr Greenwood T, Zhang Y, Wang Y, et al. Clinical presentation of hemophagocytic lymphohistiocytosis in adults is less typical than in Clinics. 2016;71:205-9.

60. Oudkerk M, Buller HR, Kuijpers D, van Es N, Oudkerk SF, McLoud TC, et al. Diagnosis, Prevention, and Treatment of Thromboembolic Complications in COVID-19: Report of the National Institute for Public Health of the Netherlands. Radiology. 2020:201629.

61. Jose RJ, Manuel A. COVID-19 cytokine storm: the interplay between inflammation and coagulation. The Lancet Respiratory medicine.

62. Guan WJ, Liang WH, Zhao Y, Liang HR, Chen ZS, Li YM, et al. Comorbidity and its impact on 1590 patients with Covid-19 in China: A Nationwide The European respiratory journal. 2020.

63. Wang LLQHDCWDHIX. Acute lung injury in patients with COVID-19 infection. Clinical and Translational Medecine. 2020;10:20-7.

64. Andrew M. Luks LF, Colin K. Grissom, Scott E. McIntosh, Robert B. Schoene, Erik R. Swenson, and Peter Hackett. COVID-19 Lung Injury is Not High Altitude Pulmonary Edema. High Altitude Medicine \& Biology. 2020:1-2.

65. Jin T, Zhu L, Bai M, He X, Wang L, Yuan D, et al. Association between the IL1R2 rs2072472 polymorphism and high-altitude pulmonary edema risk. Molecular genetics \& genomic medicine. 2019;7:e542.

66. Sharma M, Singh SB, Sarkar Genome wide expression analysis suggests perturbation of vascular homeostasis during high altitude pulmonary edema. PloS one. 2014;9:e85902.

67. Kubo K, Hanaoka M, Hayano T, Miyahara T, Hachiya T, Hayasaka M, et Inflammatory cytokines in BAL fluid and pulmonary hemodynamics in high-altitude pulmonary edema. Respiration physiology. 1998;111:301-10.

68. Alam P, Saini N, Pasha MA. MicroRNAs: An Apparent Switch for High-Altitude Pulmonary Edema. MicroRNA. 2015;4:158-67.

69. Huang C, Wang Y, Li X, Ren L, Zhao J, Hu Y, et al. Clinical features of patients infected with 2019 novel coronavirus in Wuhan, China. Lancet. 2020;395:497-506.

70. Jiang F, Yu Q, Chu Y, Zhu X, Lu W, Liu Q, et MicroRNA-98-5p inhibits proliferation and metastasis in non-small cell lung cancer by targeting TGFBR1. International journal of oncology. 2019;54:128-38. 
71. Li G, Wu F, Yang H, Deng X, Yuan Y. MiR-9-5p promotes cell growth and metastasis in non-small cell lung cancer through the repression of TGFBR2. Biomedicine \& pharmacotherapy = Biomedecine \& pharmacotherapie. 2017;96:1170-8.

72. Gaddam RR, Chambers S, Bhatia M. ACE and ACE2 in inflammation: a tale of two enzymes. Inflammation \& allergy drug targets. 2014;13:224-34.

73. Infante M, Lutman RF, Imparato S, Di Rocco M, Ceresoli GL, Torri V, et al. Differential diagnosis and management of focal ground-glass opacities. The European respiratory journal. 2009;33:821-7.

74. Zompatori M, Rimondi MR. [Diffuse ground-glass opacity of the lung. A guide to interpreting the highresolution computed tomographic (HRCT) picture]. La Radiologia medica. 1994;88:576-81.

75. Bartram U, Speer CP. The role of transforming growth factor beta in lung development and disease. Chest. 2004;125:754-65.

76. Green DR, Llambi F. Cell Death Signaling. Cold Spring Harbor perspectives in biology. 2015;7.

77. He Y, Yu D, Zhu L, Zhong S, Zhao J, Tang J. miR-149 in Human Cancer: A Systemic Review. Journal of Cancer. 2018;9:375-88.

78. Jiang L, Huang Q, Chang J, Wang E, Qiu X. MicroRNA HSA-miR-125a-5p induces apoptosis by activating p53 in lung cancer cells. Experimental lung research. 2011;37:387-98.

79. Rukov JL, Wilentzik R, Jaffe I, Vinther J, Shomron N. Pharmaco-miR: linking microRNAs and drug effects. Briefings in bioinformatics. 2014;15:648-59.

80. Magro C, Mulvey JJ, Berlin D, Nuovo G, Salvatore S, Harp J, et al. Complement associated microvascular injury and thrombosis in the pathogenesis of severe COVID-19 infection: a report of five cases. Translational research : the journal of laboratory and clinical medicine.

81. Antoniak S, Mackman Multiple roles of the coagulation protease cascade during virus infection. Blood. 2014;123:2605-13.

82. Lee N, Hui D, Wu A, Chan P, Cameron P, Joynt GM, et al. A major outbreak of severe acute respiratory syndrome in Hong Kong. The New England journal of medicine. 2003;348:1986-94.

83. Chong PY, Chui P, Ling AE, Franks TJ, Tai DY, Leo YS, et al. Analysis of deaths during the severe acute respiratory syndrome (SARS) epidemic in Singapore: challenges in determining a SARS Archives of pathology \& laboratory medicine. 2004;128:195-204.

84. Matsuo T, Kobayashi H, Kario K, Suzuki S. Fibrin D-dimer in thrombogenic disorders. Seminars in thrombosis and hemostasis. 2000;26:101-7.

85. Welty-Wolf KE, Carraway MS, Ortel TL, Piantadosi Coagulation and inflammation in acute lung injury. Thrombosis and haemostasis. 2002;88:17-25.

86. Idell S, Zwieb C, Boggaram J, Holiday D, Johnson AR, Raghu Mechanisms of fibrin formation and lysis by human lung fibroblasts: influence of TGF-beta and TNF-alpha. The American journal of physiology. 1992;263:L487-94.

87. Jablonska E, Markart P, Zakrzewicz D, Preissner KT, Wygrecka M. Transforming growth factor- beta1 induces expression of human coagulation factor XII via Smad3 and JNK signaling pathways in 
human lung fibroblasts. The Journal of biological chemistry. 2010;285:11638-51.

88. Zhao X, Nicholls JM, Chen YG. Severe acute respiratory syndrome-associated coronavirus nucleocapsid protein interacts with Smad3 and modulates transforming growth factor-beta The Journal of biological chemistry. 2008;283:3272-80.

89. Grimson A, Farh KK, Johnston WK, Garrett-Engele P, Lim LP, Bartel DP. MicroRNA targeting specificity in mammals: determinants beyond seed pairing. Molecular cell. 2007;27:91-105.

90. Kerr R, Stirling D, Ludlam CA. Interleukin 6 and haemostasis. British journal of haematology. 2001;115:3-12.

\section{Figures}

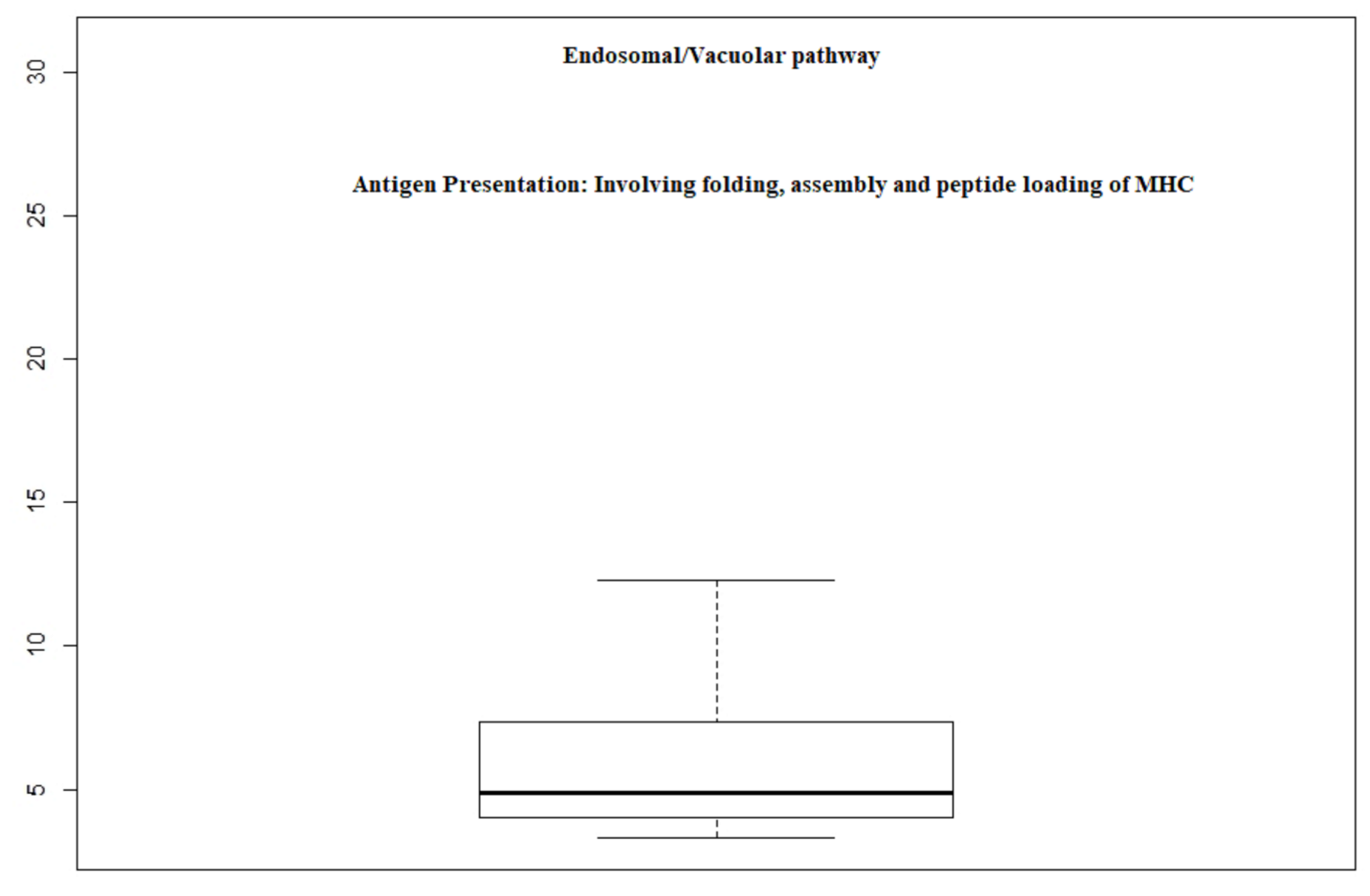

|Log(P.value)|

\section{Figure 1}

The quartile distribution of enriched pathway clusters based on |log2 (pValue)| 


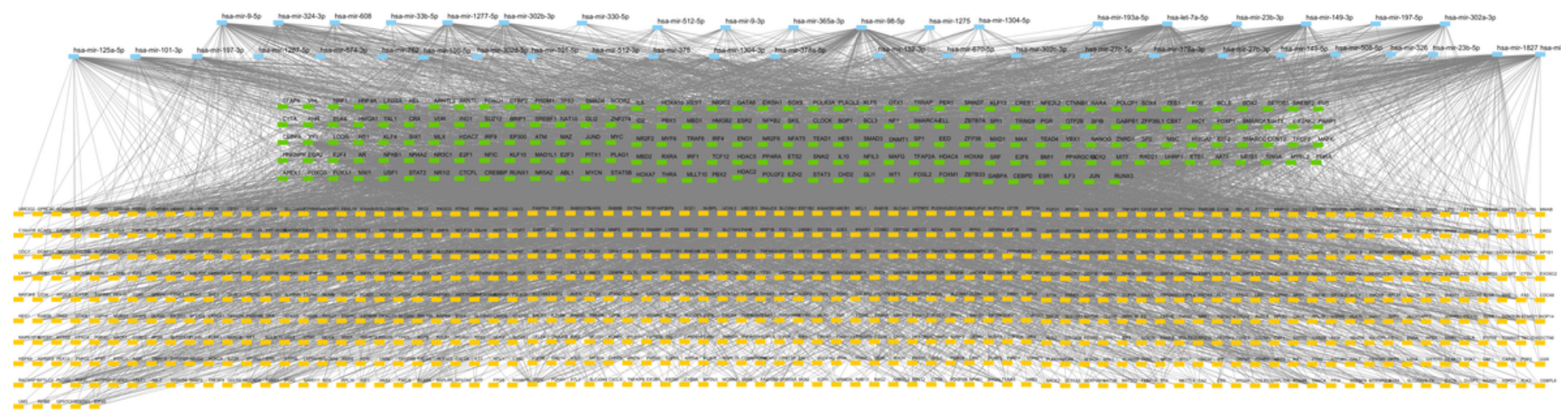

Figure 2

The FFL network identified from miRNA:TF:gene coregulatory network. The miRNAs, TFs and genes are represented in blue, green and yellow nodes respectively.

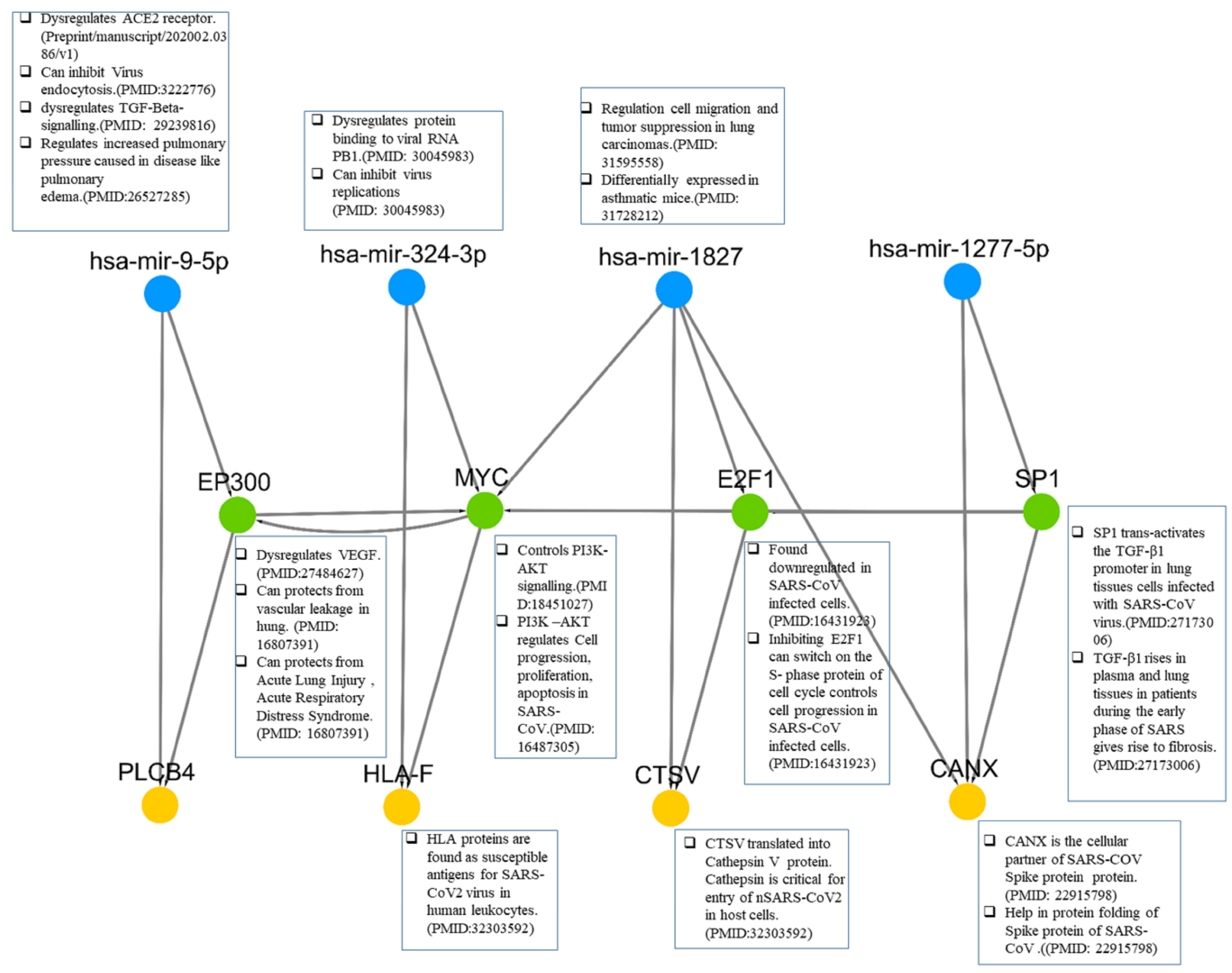

Figure 3 
The miRNA-gene regulatory circuits and interactions between them. miRNAs, TFs and genes are represented in blue, green and yellow nodes respectively. The association of each biomolecule with nSARS-CoV-2 is highlighted in boxes adjacent to them with their respective PMIDs.



\section{Figure 4}

The cohesion in the HAPE and nSARS-CoV-2 infection at both pathophysiological and molecular level with common biomolecular targets and drugs. The white boxes depicts the HAPE pathology, orange boxes depicts the common pathology of HAPE that are seen in nSARS-CoV-2 infection. Yellow boxes depicts the biomolecules from the current study which are part of SARS-CoV-2 miRNA-gene regulatory circuit or their direct interactors. The drugs used in HAPE that are proposed to be repurposed for nSARSCoV-2 are highlighted in purple text. 
Proposed targets

$\square$ Pathology for coagulation and pulmonary embolism

$\square$ Common COVID-19 pathology

$\downarrow$ inhibition

Regulation

PMID:27173006

PMID:24367666 SP1

Hsa-miR-324-3p

$3 p$

TNF- $\alpha$ TGF- $\beta$

PMID:17612493

Hsa-miR-9-5p

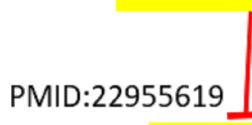

EP300
PMID:10506168

SMAD3

PMID:19853299

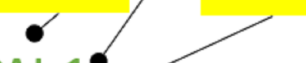

PA

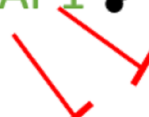

tissue plasminogen activator (tPA)

\section{Fibrinolysis}

SMAD4

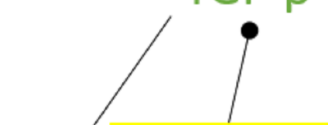

SMAD3-EP300

PMID:18055455

PA)

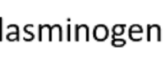

asminogen

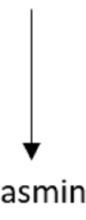



Figure 5

The similarity in the coagulation, pulmonary embolism pathology with nSARS-CoV-2 infection. The white boxes depicts the normal pathology of coagulation and pulmonary embolism, orange boxes depicts the common pathology that are seen in nSARS-CoV-2 infection. Yellow boxes depict the biomoelcules from the present study, which are part of miRNA-gene regulatory circuit in nSARS-CoV-2 or their direct regulators. The biomolecules highlighted in green (TNF-a, TGF- $\beta$ and PA-1) are proposed as main interconnections between SARS-CoV-2 and pulmonary embolism.

\section{Supplementary Files}

This is a list of supplementary files associated with this preprint. Click to download.

- SupplementaryCOVIDPAPER.pdf 